\title{
TITLE:
}

\section{High concentration crossovers of polyelectrolyte solutions}

AUTHOR(S):

Nishida, K; Kaji, K; Kanaya, T

\section{CITATION:}

Nishida, K ... [et al]. High concentration crossovers of polyelectrolyte solutions. JOURNAL OF CHEMICAL PHYSICS 2001, 114(19): 8671-8677

\section{ISSUE DATE:}

2001-05-15

URL:

http://hdl.handle.net/2433/39730

\section{RIGHT:}

Copyright 2001 American Institute of Physics. This article may be downloaded for personal use only. Any other use requires prior permission of the author and the American Institute of Physics. 


\title{
High concentration crossovers of polyelectrolyte solutions
}

\author{
K. Nishida, K. Kaji, ${ }^{a)}$ and T. Kanaya \\ Institute for Chemical Research, Kyoto University, Uji, Kyoto-fu 611-0011 Japan
}

(Received 27 October 2000; accepted 5 March 2001)

\begin{abstract}
In a few decades, several characteristic regimes and subregimes have been theoretically presented as functions of concentration $C$ and contour length for flexible polyelectrolyte solutions. However, experimentally only the dilute and semidilute regimes have been well acknowledged. In this study, solutions of sodium poly(styrene sulfonate) are studied at various concentrations ranging from the semidilute regime to the bulk through the concentrated regime using small-angle and intermediate-angle $\mathrm{x}$-ray scattering techniques. We have observed a single scattering peak being characteristic of polyelectrolyte solutions until $C=4.1 \mathrm{~mol} / \mathrm{l}$. Above $C=4.9 \mathrm{~mol} / \mathrm{l}$, such a characteristic peak disappears and instead, another new peak appears at higher $q$ 's. In the plot of the maximum position $q_{\max }$ vs $C$, two crossover concentrations are found at $C^{* *}=1.2 \mathrm{~mol} / \mathrm{l}$ and $C^{* * *}=4.5 \mathrm{~mol} / \mathrm{l}$. The former is a continuous crossover from $q_{\max } \sim C^{1 / 2}$ to $q_{\max } \sim C^{1 / 4}$ which can be assigned to the crossover of the semidilute-to-concentrated regime, while the latter is a discontinuous crossover which may be assigned to that of the concentrated-to-swollen regime. In the swollen regime the characteristics of polyelectrolyte solutions are lost. The discontinuous crossover concentration $C^{* * *}$ is about half the bulk concentration $C_{\text {bulk }}=8.4 \mathrm{~mol} / \mathrm{l}$. (C) 2001 American Institute of Physics. [DOI: 10.1063/1.1367383]
\end{abstract}

\section{INTRODUCTION}

Polyelectrolyte solutions without added salts are generally considered to assume three regimes, dilute, semidilute, and concentrated, as in the case of neutral polymer solutions. However, intramolecular and intermolecular electrostatic repulsive forces complicatedly affect the crossover concentrations between them depending on concentration or ionic strength. Dilution causes the polyion chains to expand; it increases their persistence lengths as well as their excluded volumes. Such chain expansion shifts the crossover concentrations greatly towards lower concentrations compared with those for neutral polymers. Furthermore, intermolecular or intersegmental forces give some order to the polyelectrolyte solution. The efforts to understand these features quantitatively have been made for half a century, but our understanding is still insufficient.

Theoretically, de Gennes et al. ${ }^{1}$ studied the possibility of a three-dimensional periodic lattice (bcc) model for the dilute regime, assuming pointlike polyions as constituents. However, they commented in the same paper that the possibility of such a lattice structure is very rare and suggested a liquidlike ordering structure, i.e., the closest packing of spherical particles of polyions having a radius shorter than the screening length. On the other hand, they proposed an isotropic model for the semidilute regime after examining the possibilities of a hexagonal lattice model and a cubic lattice model, both consisting of rigid rod polyions. In the isotropic model, polyion chains are entangled and behave ideally as a whole, but they are nearly stiff within a correlation length $\xi$ of the chain overlap size: $\xi \sim b_{t}$ where $b_{t}$ is persistence length. These structure models predict that a

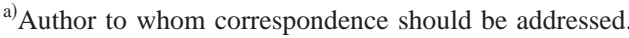

single broad peak appears in the scattering function and that the concentration $C$ dependencies of the peak position $q_{\max }$ are given as $q_{\max } \sim(C / N)^{1 / 3}$ and $q_{\max } \sim C^{1 / 2}$ for the dilute and semidilute regimes, respectively.

Later, Odijk ${ }^{2}$ suggested that the semidilute regime was separated into three subregimes depending on the concentration: lattice subregime for $\xi<L<b_{t}$, transition subregime for $\xi<b_{t}<L$ and blob subregime for $\xi<b_{t}$, where $L$ is polyion contour length. However, these subregimes are questionable because the concentration dependence of electrostatic persistence length employed by this author hardly agrees with observed data. ${ }^{3-7}$

Thereafter, the semidilute-to-concentrated crossover was discussed by Joanny and Leibler, ${ }^{8}$ Vilgis and Borsali, ${ }^{9}$ Barrat and Joanny, ${ }^{10}$ and Muthukumar. ${ }^{11}$ All of these authors have given a relation $q_{\max } \sim C^{1 / 4}$ for the concentrated regime, but the semidilute-to-concentrated crossover concentrations are greatly different from author to author.

Experimentally the efforts to confirm the predictions by de Gennes et al. ${ }^{1}$ and Odijk $^{2}$ were made by many authors. ${ }^{12-21}$ Nierlich et al. ${ }^{12}$ found the relation $q_{\max } \sim C^{1 / 2}$ for the semidilute region for the first time using a small angle neutron scattering (SANS) technique. Later, Kaji et al. ${ }^{13}$ investigated the concentration dependencies of $q_{\max }$ more precisely using a small angle x-ray scattering (SAXS) technique, which made it possible to determine the crossover concentration $C^{*}$ from the dilute regime $\left(q_{\max } \sim(C / N)^{1 / 3}\right)$ to the semidilute regime $\left(q_{\max } \sim C^{1 / 2}\right)$. These relations were also confirmed by Krause et al. ${ }^{14}$ and Johner et al. ${ }^{15}$ using a light scattering technique. Dynamic measurements by a quasielastic neutron scattering technique ${ }^{16}$ and a high frequency dielectric relaxation method ${ }^{17}$ supported such crossover concentrations as well.

This $C^{*}$ is usually assumed to be the boundary between 
the dilute and semidilute regime. We, on the other hand, calculated the concentration of the radius-of-gyration contact, $C_{S}^{*}$, as a function of molecular weight using Le Bret's persistence length. $^{22} \mathrm{We}$ actually confirmed ${ }^{13}$ that the $C^{*}$ corresponds well to the $C_{S}^{*}$ for NaPSS with weight-average molecular weights $M_{w}$ ranging from 8000 to 100000 . This correspondence $\left(C^{*} \approx C_{S}^{*}\right)$ is valid when the degree of polymerization is not so high $\left(N<10^{3}\right.$, see note $\left.{ }^{23}\right)$. Thus, the problem of the boundary between the dilute and semidilute regime has become considerably clear. However, the information is still lacking to select the most appropriate model for the dilute regime and to judge the existence of the subregimes within the semidilute regime, because $q_{\max }$ $\sim(C / N)^{1 / 3}$ relation for the dilute regime is valid for the liquidlike ordering as well as the three dimensional periodic lattice model, and $q_{\max } \sim C^{1 / 2}$ for semidilute regime is valid for the isotropic model as well as the lattice and the transition subregimes. As for the dilute regime, the appearance of the periodic lattice seems to be unlikely also from an experimental viewpoint, since no higher order peak has been detected in the scattering experiments of the dilute regime. ${ }^{14}$ Concerning the semidilute regime, no obvious evidences for the inter-subregime distinction have yet been detected experimentally.

Thus, the actual classification of regimes in polyelectrolyte solutions seems rather simple contrary to the theoretical expectation. Is there any experimentally detectable transition other than the dilute-semidilute transition? In this study, we report and discuss $\mathrm{x}$-ray scattering data obtained about polyelectrolyte solutions in the semidilute to the bulk state. It is the main object of this work to explore whether the structure of polyelectrolyte solutions in the semidilute regime is qualitatively universal up to a highly concentrated state (several mol/1). To our knowledge, structures of such highly concentrated polyelectrolyte solutions have not been studied using scattering methods.

\section{EXPERIMENT}

\section{A. Materials}

A sodium poly(styrene sulfonate) (NaPSS) was prepared from polystyrene (PS) by a conventional method. ${ }^{24}$ The parent PS with a degree of polymerization $N \approx 1200$ was purchased from Nacalai Tesque, Inc. The degree of sulfonation was determined to be more than 0.99 , by a test of the integrated spectra of ${ }^{1} \mathrm{H}-\mathrm{NMR}$. Polyelectrolyte concentrations were varied from $0.1 \mathrm{~mol} / 1$ to bulk state. The concentration for the bulk state, $C_{\text {bulk }}$, was estimated to be $8.4 \mathrm{~mol} / \mathrm{l}$ via density measurements. Deionized water was used as the solvent and no external salts were added.

\section{B. X-ray scattering measurements}

The x-ray scattering measurements were carried out at $25^{\circ} \mathrm{C}$ using a $6 \mathrm{~m}$ point focusing SAXS camera at the highintensity X-ray Laboratory of Kyoto University. ${ }^{25}$ Two instrumental configurations for $\mathrm{Cu} K \alpha$ radiation were selected depending on $C$ because the $q$ range expected for the characteristic maximum would change considerably with $C$; the camera distances were employed to be 1.6 and $0.6 \mathrm{~m}$ for $C$

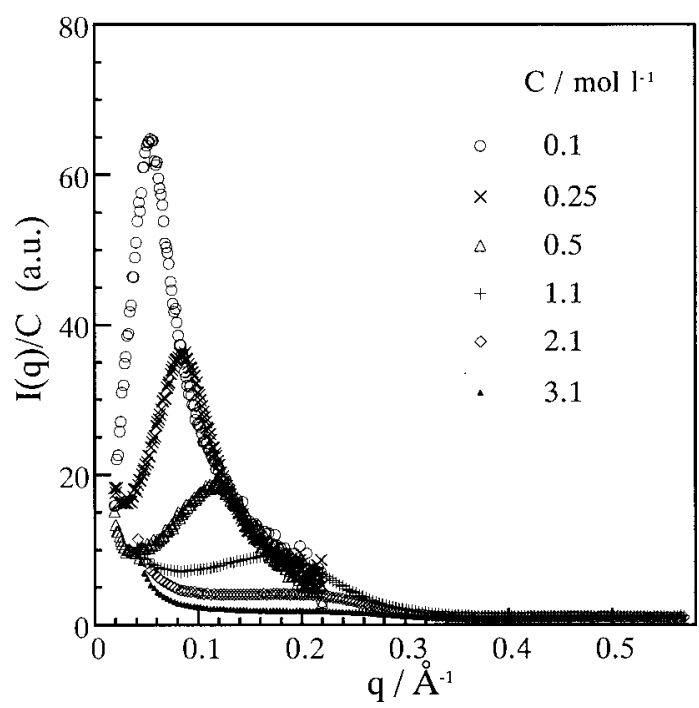

FIG. 1. Concentration dependence of x-ray scattering curves for NaPSS solutions. Concentrations are shown in the figure.

$=0.1-1.1 \mathrm{~mol} / 1$ and $C=2.1 \mathrm{~mol} / \mathrm{l}-$ bulk state, respectively, alternatively corresponding to $q=0.02-0.2 \AA^{-1}$ and $q$ $=0.04-0.57 \AA^{-1}$, respectively. $q$ is the length of scattering vector $q=4 \pi \sin \theta / \lambda, 2 \theta$ and $\lambda$ being the scattering angle and $x$-ray wavelength, respectively. The scattering intensities from all the sample solutions at various concentrations examined did not show any anisotropy on the two-dimensional detector, so the intensity was radially averaged. Then, the scattering intensity from the solvent was subtracted and the excess intensity, $I(q)$, was normalized by $C$.

\section{RESULTS}

\section{A. Concentration dependence of scattering curves}

All the scattering intensity curves normalized by concentration, $I(q) / C$, are shown as a function of $C$ by dividing into four concentration regions, $C=0.1-3.1 \mathrm{~mol} / 1,3.1-5.6$ $\mathrm{mol} / \mathrm{l}, 5.6-7.4 \mathrm{~mol} / \mathrm{l}$, and $7.7 \mathrm{~mol} / \mathrm{l}-$ bulk in Figs. 1 to 4 , respectively.

In Fig. 1 for the lowest concentrations a well-known single characteristic peak of polyelectrolyte solutions is observed, which is understood as a correlation peak due to strong electrostatic repulsion between polyion segments. With increasing $C$ the peak position, $q_{\max }$, shifts to higher scattering angles and the maximum intensity decreases, meaning that both the correlation length and strength decrease with increasing $C$. The latter effect is due to the increase of the counterion concentration or ionic strength. At $C=3.1 \mathrm{~mol} / 1$ the scattering peak almost disappears in this scale, and its curve looks monotonous. However, when enlarged, it still indicates a broad peak or a shoulder as seen from Fig. 2.

Figure 2 shows $I(q) / C$ for $C=3.1 \sim 5.6 \mathrm{~mol} / \mathrm{l}$ where the scale of the ordinate is one order of magnitude enlarged from Fig. 1. Even at such concentrations, a trace of the polyelec- 


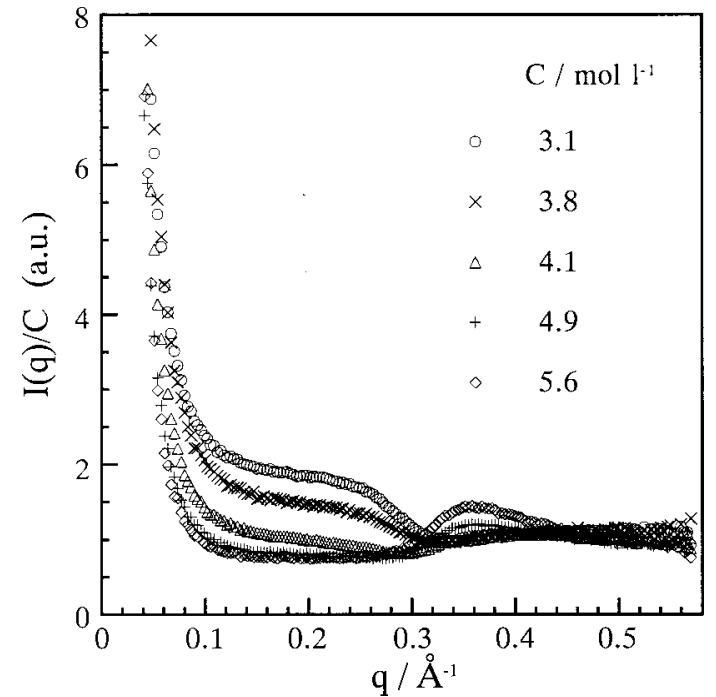

FIG. 2. Concentration dependence of x-ray scattering curves for NaPSS solutions. Concentrations are shown in the figure.

trolyte peak is still observed at around $q=0.25 \AA^{-1}$ until $C=4.1 \mathrm{~mol} / \mathrm{l}$. However, above $4.9 \mathrm{~mol} / \mathrm{l}$ this peak disappears completely and another new peak appears at around $q$ $=0.35 \AA^{-1}$. The intensity of the new peak is at first very weak and $q_{\max }$ scarcely moves with $C$. Judging from these observations, the new peak may be considered different from the characteristic peak of polyelectrolyte solutions. As the concentration further increases, this new peak becomes increasingly intense, and $q_{\max }$ slightly moves toward higher $q$ 's (see Fig. 3 for $C=5.6-7.4 \mathrm{~mol} / \mathrm{l}$ ). Even at extremely high concentrations, $C=7.7-8.4 \mathrm{~mol} / 1$ (bulk), $q_{\max }$ still continues to slightly shift toward higher $q$ 's as shown in Fig. 4; the final $q_{\text {max }}$ value is $0.39 \AA^{-1}$. On the other hand, the peak intensity has a maximum at $7.7 \mathrm{~mol} / \mathrm{l}$ and then it begins to decrease while the peak slightly becomes broad with $C$ in this concentration range. This may be because the distribution of intermolecular distances is broadened due to the partial contact of molecules. The scattering peak in the bulk

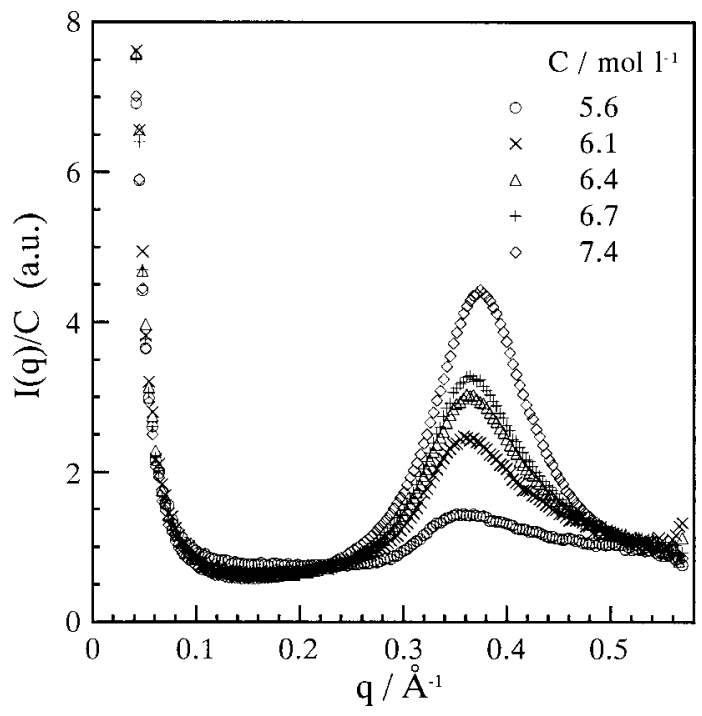

FIG. 3. Concentration dependence of x-ray scattering curves for NaPSS solutions. Concentrations are shown in the figure.

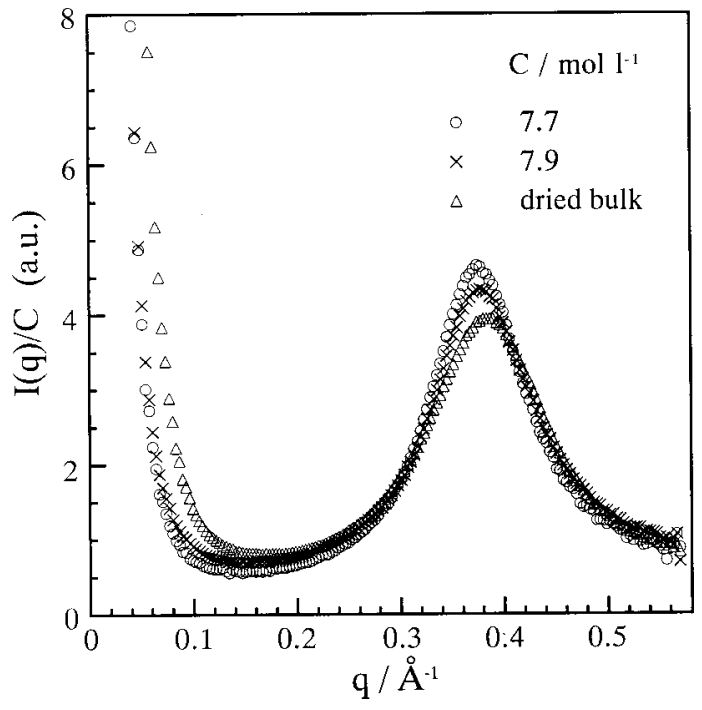

FIG. 4. Concentration dependence of x-ray scattering curves for NaPSS solutions. Concentrations are shown in the figure.

state is undoubtedly assigned to the so-called amorphous halo due to the average intermolecular distance.

In order to make clear the difference in the scattering curves between neutral polymer and polyelectrolyte solutions, preliminary measurements for toluene solutions of $a$-PS have been conducted; no literature results were found. The results were as expected though the observed scattering curves are not reproduced here. Generally, the total scattering intensity of a polymer solution is given as the sum of intramolecular and intermolecular correlation contributions. In the bulk state of $a$-PS, which corresponds to $C$ $=10.05 \mathrm{~mol} / \mathrm{l}$, where intermolecular correlations are dominant, the scattering curve indicated the well-known amorphous halo, whose peak position $\left(q_{\max }=0.65 \AA^{-1}\right)$ corresponds to the average intermolecular distance of $9.7 \AA$. As the system was swollen and further diluted, the intermolecular distances increased with losing the intermolecular correlations, resulting that the amorphous halo became broader and weaker. Instead, the intensities at low $q$ 's increased with dilution because of the increase of the contribution from intramolecular correlations. At the infinite dilution limit the scattering curve should naturally show a scattering function of a single chain, a decreasing function having a maximum at $q=0$.

As was shown before, the polyelectrolyte solutions also indicated the intermolecular peak in the bulk state and it became broader and weaker with swelling and further dilution. In this case, however, another peak due to the intermolecular electrostatic repulsive forces appeared at a lower $q$ and increased in intensity with further decreasing concentration. Thus, the significant point is that in the case of neutral polymer solutions "no polyelectrolyte peak" grows in further dilution.

\section{B. Concentration dependence of the peak position and the peak intensity}

In Fig. 5 all the peak positions $q_{\max }$ of the measured scattering curves in Figs. 1-4 are plotted as a function of 


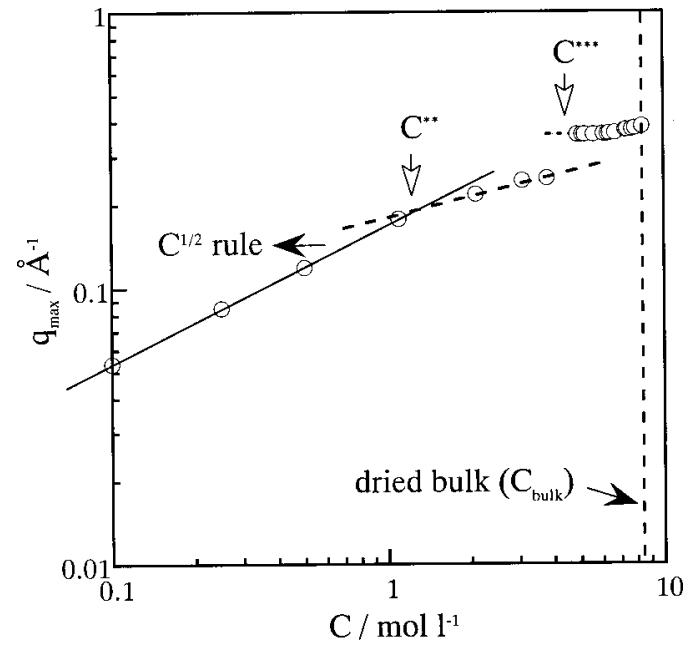

FIG. 5. $q_{\max }$ vs $C$ plot in logarithmic scales for NaPSS solutions. A solid line represents a slope of 1/2. A broken line represents a slope of 1/4.

concentration $C$. In this figure two transition concentrations $C^{* *}$ and $C^{* * *}$ are clearly seen. The first one, $C^{* *}$ $=1.2 \mathrm{~mol} / \mathrm{l}$, can be assigned to the crossover concentration of the semidilute regime to the concentrated regime; below $C^{* *}$ the well-known rule $q_{\max } \sim C^{1 / 2}$ for the semidilute regime holds while above $C^{* *}$ a relation $q_{\max } \sim C^{1 / 4}$, theoretically predicted for the concentrated regime, is obtained. Wang et ll $^{26}$ have also reported a characteristic concentration $(C \approx 1.0 \mathrm{~mol} / \mathrm{l})$ where the concentration dependence of the reduced osmotic pressure $(\Pi / R T)$ of NaPSS solutions changes drastically. They mentioned this behavior as a recovery to a random coil conformation like in solution of neutral polymers. This crossover concentration will closely be discussed later. The second transition, ${ }^{27} C^{* * *}$ $=4.5 \mathrm{~mol} / \mathrm{l}$, may be considered the crossover concentration from the concentrated to the swollen regime which is no longer a solution. Thus, there exists a discontinuous gap in $q_{\max }$ at around $C^{* * *}$ and the peaks below and above $C^{* * *}$ are essentially different in nature. Figure 6 shows the peak intensity normalized by concentration, $I\left(q_{\max }\right) / C$, as a func-

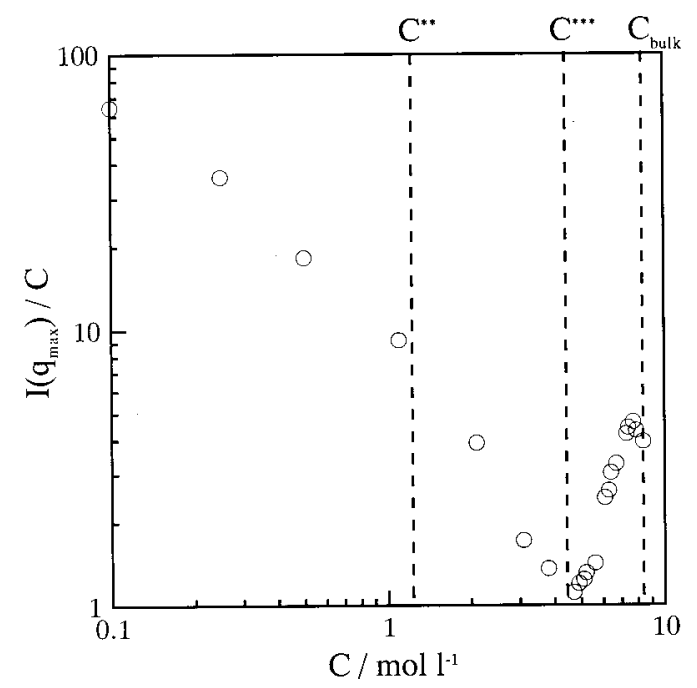

FIG. 6. Comparison of calculated Debye length between two models. tion of concentration. As described before and also seen from this figure, in the concentrated regime for $C<C^{* * * *}$ the peak intensity decreases with increasing $C$ whereas in the swollen regime for $C>C^{* * *}$ the peak intensity increases with $C$ except at highest concentrations.

\section{DISCUSSION}

In this study we have experimentally found the two crossover concentrations, the semidilute-to-concentrated regime $C^{* *}$ and the concentrated-to-swollen regime $C^{* * *}$; the former is a smooth transition and the latter is a discontinuous one. Here, the former crossover concentration is first discussed quantitatively in comparison with the theories, and then some comments will be made.

\section{A. Semidilute-to-concentrated crossover concentration}

Here, the former crossover concentration, $C^{* *}$, is quantitatively discussed based on some proposed theories. Joanny and Leibler, ${ }^{8}$ Vilgis and Borsali, ${ }^{9}$ Barrat and Joanny, ${ }^{10}$ and Muthukumar $^{11}$ gave a relation $q_{\max } \sim C^{1 / 4}$ for the concentrated regime, and an equation for the crossover concentration between the semidilute and the concentrated regime. The $q_{\text {max }} \sim C^{1 / 4}$ relation agrees well with the observed one as was mentioned in the section of Results. However, the observed crossover concentration $C^{* *}$ of the semidilute to concentrated regime does not agree well with the theoretical predictions. Here, we derive a numerical value for $C^{* *}$ along the way of Muthukumar's renormalization treatment ${ }^{11}$ of these theories. Assuming that the correlation lengths for the two regimes should agree with each other at a crossover concentration though theoretically the crossover occurs gradually, the theoretical crossover concentration $C_{\mathrm{MU}}^{* *}$ is obtained as

$$
C_{\mathrm{MU}}^{* *}=0.79\left(\omega_{c} / \ell\right)^{1 / 3} / \ell^{3},
$$

where $\ell$ is Kuhn length and the strength of Coulomb potential

$$
\omega_{c}=4 \pi Z_{p}^{2} e^{2} \alpha^{2} / K_{r} \varepsilon_{0} k_{B} T,
$$

$Z_{p}, e, \alpha, K_{r}, \varepsilon_{0}, k_{B}$, and $T$ being the number of charges per segment, the electronic charge, the degree of ionization per chain, the relative dielectric constant of the medium, the absolute dielectric constant of the vacuum, Boltzmann constant and temperature, respectively. In the present case $Z_{p}=1, \alpha$ $=0.35, K_{r}=78.4$ for water at $25^{\circ} \mathrm{C}$ and $T=298 \mathrm{~K}$, resulting in $\omega_{c}=138.3 \AA$. When the persistence length of nonionized NaPSS, $b_{0}$, is taken to be $12 \AA,{ }^{20}$ we obtain $\ell=24 \AA$ and then $C_{\mathrm{MU}}^{* *}=0.17 \mathrm{~mol} / \mathrm{l}$. This value is one order of magnitude lower than the observed one of $1.2 \mathrm{~mol} / \mathrm{l}$. As is expected from Eq. (1), $C_{\mathrm{MU}}^{* *}$ is strongly dependent on the value of $\ell$. The estimation of $\ell$ causes a great change in $C_{\mathrm{MU}}^{* *}$; the difference by a factor in $\ell$ is amplified to that by one order of magnitude in $C_{\mathrm{MU}}^{* *}$. Therefore, we reconsidered the value of $b_{0}$. We independently reported concentration dependence of the persistence length of NaPSS. Using our original data, ${ }^{3}$ the observed value of persistence length, $b_{t}$, were extrapolated to $C^{-1 / 2}=0$ to obtain $b_{0}=9 \pm 2 \AA$ or $\ell=18 \pm 4 \AA$. The value $\ell=18 \AA$ gives $C_{\mathrm{MU}}^{* *}=0.44 \mathrm{~mol} / \mathrm{l}$. The agreement with 


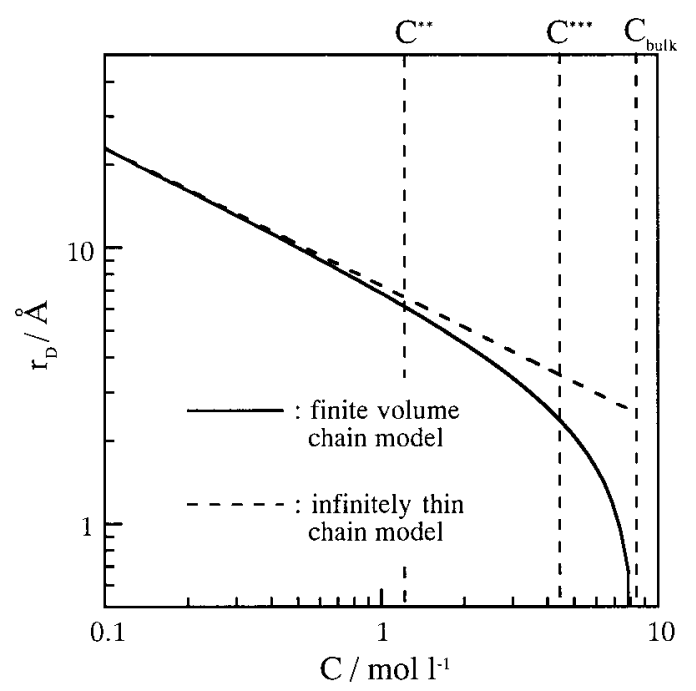

FIG. 7. Concentration dependence of the peak intensity normalized by concentration, $I\left(q_{\max }\right) / C$.

the observed $C^{* *}$ has been greatly improved. If we employ $b_{0}=7 \AA$ or $\ell=14 \AA$ as the lower limit, we obtain $C_{\mathrm{MU}}^{* *}$ $=1.03 \mathrm{~mol} / \mathrm{l}$, which is very close to the observed $C^{* *}$, but there is no reason for doing so. Barrat and Joanny ${ }^{10}$ also gave the theoretical crossover concentration based on the electrostatic blob concept that the electrostatic blob size $\xi_{e}$ is nearly equal to the mesh size $\xi$ of the isotropic structure in the semidilute regime. However, their crossover concentration seems considerably high in more than two orders of magnitude compared with observed value. One reason for this discrepancy may be due to the used theory for the concentration dependence of persistence length. ${ }^{2}$

\section{B. Calculation of Debye length}

In order to understand the electrostatic screening effects on the structure of polyelectrolyte solutions we have calculated the electrostatic interactions as a function of concentration. Figure 7 shows the calculated Debye screening length, $r_{D}$, as a function of $C$, assuming the following two models. One is "an infinitely thin chain model" where shielding counterions are freely distributed without being influenced by the specific volume of polyions themselves. The other is "a finite volume chain model" where shielding ions are distributed in the effective space from which the specific volumes of the polyions are excluded. In both cases, the proportion of uncondensed counterions to the total ones is taken as $35 \%$ based on the condensation theory by Oosawa ${ }^{28}$ and Manning, ${ }^{29}$ and these uncondensed counterions are assumed to contribute to the Debye-type screening effect, and a reduction in the degree of dissociation is not taken into consideration. In the dilute limit, the calculated $r_{D}$ values for both models almost agree with each other. With increasing concentration, the infinitely thin chain model follows the relationship, $r_{D} \sim C^{-1 / 2}$, meaning that the distance of electrostatic interactions is shortened in the same manner as the inter-segmental distance, $\xi \sim C^{-1 / 2}$ in the semidilute regime. If this were the case, self-similarity of the structure might be

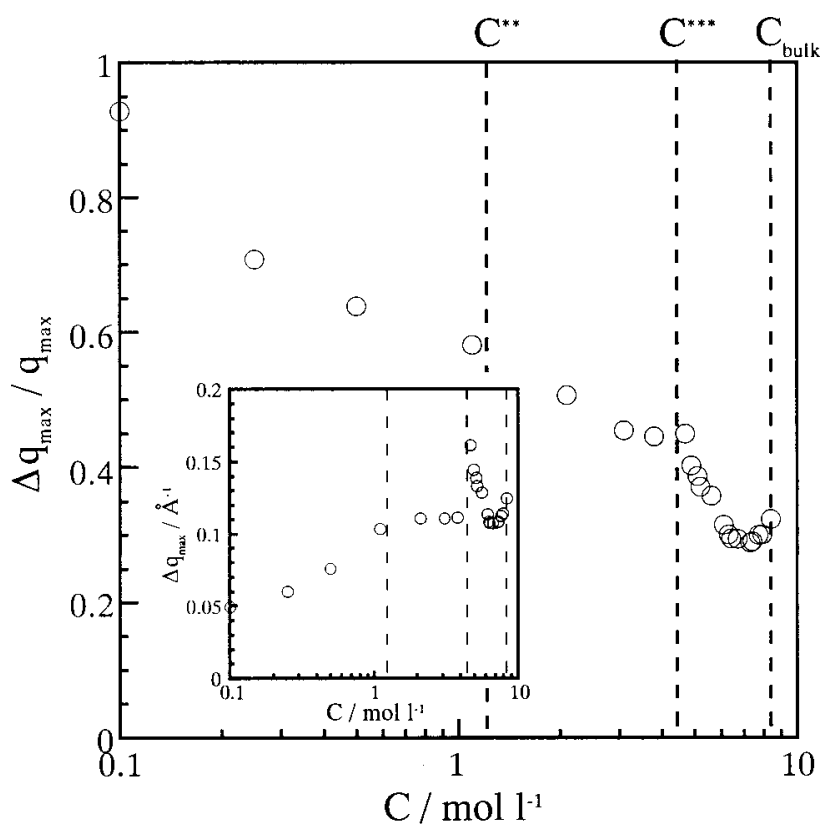

FIG. 8. Normalized peak width, $\Delta q_{\max } / q_{\max }$, vs $C$ plot in semilogarithmic scale for NaPSS solutions. The inset shows the peak width without normalization by $q_{\max }$.

maintained and hence the semidilute regime might possibly extend until the bulk state. In the finite volume chain model, however, $r_{D}$ decreases more rapidly than the relationship $r_{D} \sim C^{-1 / 2}$ as shown in Fig. 7. This finite volume chain model, needless to say, is more realistic rather than the infinitely thin chain model, especially in the concentrated region. In the actual solutions, polymer chains already occupy approximately half the total volume of the solution at around $C=4 \mathrm{~mol} / \mathrm{l}$. Thus, the density of shielding ions increases drastically, and accordingly $r_{D}$ also decreases significantly. Once a critical concentration is exceeded, above which the electrostatic repulsive forces are suppressed considerably and the van der Waals attractive forces surpass the electrostatic repulsive forces, the segments are allowed to approach the closest separation as in the bulk state. This may cause the swollen state.

\section{Abnormal scattering behavior in the swollen regime}

What is interesting in the swollen regime, $C>C^{* * *}$, is the behavior of SAXS curves for concentrations above 7.7 mol/l, which is shown in Fig. 4. The peak in this concentration range decreases in intensity (also see Fig. 6) and increases in width with concentration. This behavior seems peculiar because it is contrary to the usual expectation that the distribution of intermolecular distance is broadened with degree of swelling. In order to examine such behavior closely, the peak width normalized by the peak position, $\Delta q_{\max } / q_{\max }$, are also plotted as a function of $C$ in Fig. 8. The value $\Delta q_{\max } / q_{\max }$ can be used as an index of structural fluctuations relative to the size of the structure. In the beginning this value decreases monotonously with increasing concentration until $7.7 \mathrm{~mol} / \mathrm{l}$ as expected whereas above $7.7 \mathrm{~mol} / \mathrm{l}$ the width of the peak increases with increasing $C$. These 
experimental results suggest that above $7.7 \mathrm{~mol} / \mathrm{l}$ the molecular motions begin to be restricted by partial inter-segmental contact and hence the symmetry of the segments around the chain axis begins to be destroyed.

\section{Concentration dependence of persistence length and subregimes}

Finally, we will discuss the concentration dependence of persistence length, which is one of the most important relations for polyelectrolyte solutions, and the relations with the subregimes in the semidilute regime.

Several subtransitions were predicted to take place in the semidilute regime, as was mentioned in the introduction. This would be true if the relationships among the characteristic parameters $\left(\xi, L, b_{t}\right)$ meet the conditions mentioned in the Introduction. Despite the recent intensive studies, obvious evidence for such subtransitions has not been found. The most suspicious point of realization of such conditions is the concentration behavior of the persistence length, $b_{t}$, especially its electrostatic part, $b_{e}$. Here it is assumed that $b_{t}$ and $b_{e}$ are related by an equation $b_{t}=b_{e}+b_{0}, b_{0}$ being a bare persistence length intrinsic to the nonionized backbone chain. The electrostatic persistence length, $b_{e}$, is mainly ruled by the screening effects if the charge density is constant. Intuitively this effect leads to the nature that $b_{e}$ decreases with increasing polyelectrolyte concentration $C$ or more generally ionic strength, $I$. Recently this relation has been discussed quantitatively using a power law type equation, $b_{e} \sim C^{-\alpha}$. Roughly speaking, two types of theories are reported, i.e., $b_{e} \sim C^{-1}$ relation $^{22,30-32}$ and $b_{e} \sim C^{-1 / 2}$ relation. ${ }^{22,32-34}$ However, the majority of experimental data support not only the $b_{e} \sim C^{-1 / 2}$ relation, ${ }^{3-7}$ but also the relation between the inter-segmental correlation length $\xi$ and concentration, $\xi \sim C^{-1 / 2}$ as long as the concentration is in the semidilute regime, $C^{*}<C<C^{* *}(C<1.2 \mathrm{~mol} / \mathrm{l})$. This means that both the electrostatic persistence length and the inter-segmental correlation length have a parallel relationship, $b_{e} \sim \xi \sim C^{-1 / 2}$. This impressive coincidence is one of evidences for the validity of the isotropic model proposed by de Gennes et al. ${ }^{1}$ On the other hand, the nematic or the lattice subregime and the transition subregime predicted by Odijk $^{2}$ also have the relation $\xi \sim C^{-1 / 2}$, but in these cases the conditions $b_{t} \gg \xi$ and $b_{t}>\xi$, should be satisfied, respectively, and hence these subregimes cannot be realized as Barrat and Joanny also predicted. ${ }^{10}$ Therefore, we can conclude that the structure in the semidilute regime is represented by the isotropic model proposed by de Gennes et al. ${ }^{1}$ at any condition.

\section{E. Upturn in low-q range}

Upturn of the scattering in the low- $q$ range is generally considered caused by both dynamical fluctuations and static inhomogeneity. The low- $q$ upturn of the typical polyelectrolyte solution is usually attributed to the dynamical fluctuations, though there exists still controversy about the origin of it. ${ }^{35}$ Theoretically, the structure factor $S(0)$ is related to the density fluctuations due to the isothermal compressibility $\chi_{T}: 1,36$

$S(0) \sim k_{B} T \chi_{T}$.

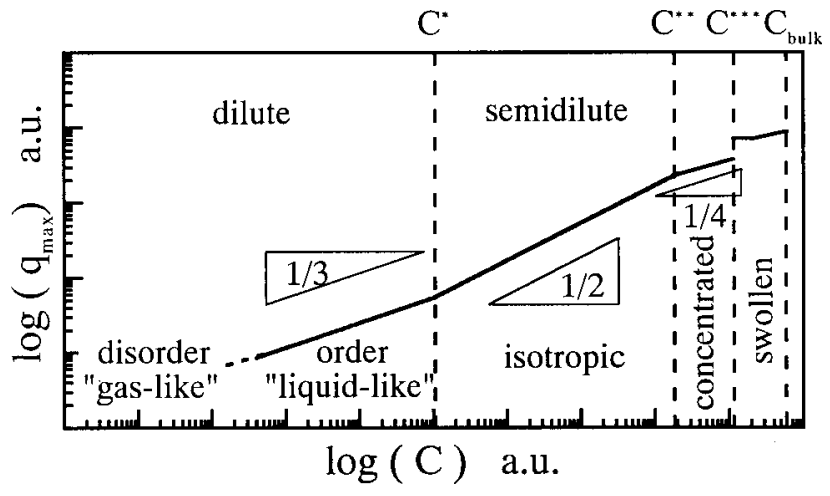

FIG. 9. Schematic of $q_{\max }-C$ plot in logarithmic scales for NaPSS solutions.

Unfortunately, we cannot obtain the quantity $S(0)$ from the present experiments. What is measured is the scattering intensity $I(q)$, and the extrapolated quantity $I(q \rightarrow 0)$ seems to somewhat reflect the dynamical fluctuations in polyionpolyion structure, however, it is strongly influenced by the form factor of polyion $P(q)$ from the relation: ${ }^{35}$

$$
S(q) \sim I(q) / P(q) .
$$

In order to obtain such $P(q)$, neutron scattering experiments with an elaborate labeling method is indispensable. ${ }^{20}$ This is out of the scope of the present work.

On the other hand, the low- $q$ upturn of the bulk polymer is usually attributed to the static inhomogeneity. Recently, such inhomogeneity in the bulk (glass) polymer has been extensively studied in terms of the so-called 'Fischer's cluster.', 37

Under these circumstances, the discussion for the upturn in the low- $q$ range is a topic of controversy, and hence it is rather speculative. At present, we presume that the upturn appearing in a $q$ range lower than the polyelectrolyte peak (see Fig. 1) is caused from the dynamical reason, whereas the upturn in the low- $q$ range after the polyelectrolyte peak disappeared (see Fig. 4) is the static inhomogeneity. The upturn in the intermediate concentrations between Figs. 1 and 4 is supposed to originate complicatedly from dynamical and static reasons.

It may safely be said that the upturn in the low- $q$ range for the dried bulk is more remarkable than the slightly swollen NaPSS as is seen in Fig. 4. This fact shows that dried bulk is more inhomogeneous than the slightly swollen polymer. This interpretation seems to be consistent with the discussion as described in Sec. IV C.

\section{CONCLUSIONS}

The structure of NaPSS solution in the semidilute regime is qualitatively universal up to $1.2 \mathrm{~mol} / \mathrm{l}$, and the whole semidilute regime seems to fall within the isotropic model proposed by de Gennes et al. ${ }^{1}$ Above this concentration, the solution enters the concentrated regime, where the electrostatic interaction begins to be weakened more rapidly. At last, the electrostatic repulsion is surpassed by the van der Waals attraction at around $4.5 \mathrm{~mol} / \mathrm{l}$, and then the structure suddenly turns into the swollen regime. Finally, the relation- 
ship between the position of the scattering maximum, $q_{\max }$, and the concentration of NaPSS, $C$, is summarized schematically in Fig. 9 for the readers' convenience. In mean future, we will report a modified phase diagram for the actual system based on the recently accumulated reliable data.

\section{ACKNOWLEDGMENTS}

This study was carried out using the 6-m point focusing SAXS camera at the High-Intensity X-ray Laboratory of Kyoto University (KUHIXL). We thank the KUHIXL committee. We are indebted to Professor S. Suehiro for the kind help of the data processing. We thank Professor J.-F. Joanny, Professor R. Borsali, and Professor M. Muthukumar for fruitful discussions.

${ }^{1}$ P. G. de Gennes, P. Pincus, R. M. Velasco, and F. Brochard, J. Phys. (France) 37, 1461 (1976).

${ }^{2}$ T. Odijk, Macromolecules 12, 688 (1979).

${ }^{3}$ K. Nishida, H. Urakawa, K. Kaji, B. Gabrys, and J. S. Higgins, Polymer 38, 6083 (1997).

${ }^{4}$ A. Takahashi, T. Kato, and M. Nagasawa, J. Phys. Chem. 71, 2001 (1967).

${ }^{5}$ S. Förster, M. Schmidt, and M. Antonietti, J. Phys. Chem. 96, 4008 (1992).

${ }^{6}$ V. Degiorgio, F. Mantegazza, and R. Piazza, Europhys. Lett. 15, 75 (1991).

${ }^{7}$ M. Tricot, Macromolecules 17, 1698 (1984).

${ }^{8}$ J.-F. Joanny and L. Leibler, J. Phys. (France) 51, 545 (1990).

${ }^{9}$ T. A. Vilgis and R. Borsali, Phys. Rev. A 43, 6857 (1991).

${ }^{10}$ J.-L. Barrat and J.-F. Joanny, Adv. Chem. Phys. XCIV, 1 (1996).

${ }^{11}$ M. Muthukumar, J. Chem. Phys. 105, 5183 (1996).

${ }^{12}$ M. Nierlich, C. E. Williams, F. Boué, P. J. Cotton, M. Daoud, B. Farnoux, G. Jannink, C. Picot, M. Moan, C. Wolff, M. Rinaudo, and P. G. de Gennes, J. Phys. (France) 40, 701 (1979).

${ }^{13}$ K. Kaji, H. Urakawa, T. Kanaya, and R. Kitamaru, J. Phys. (France) 49, 993 (1988).

${ }^{14}$ R. Krause, E. E. Maier, M. Deggelmann, M. Hagenbüchle, S. F. Schulz, and R. Weber, Physica A 160, 135 (1989).

${ }^{15}$ C. Johner, H. Kramer, S. Batzill, C. Graf, M. Hagenbüchle, C. Martin, and R. Weber, J. Phys. II 4, 1571 (1994).

${ }^{16}$ T. Kanaya, K. Kaji, R. Kitamaru, J. S. Higgins, and B. Farago, Macromolecules 22, 1356 (1989).

${ }^{17}$ K. Ito, A. Yagi, N. Ookubo, and R. Hayakawa, Rep. Prog. Polym. Phys. Jpn. 29, 87 (1986).
${ }^{18}$ C. E. Williams, M. Nielich, P. J. Cotton, G. Jannink, F. Boué, M. Daoud, B. Farnoux, C. Picot, P. G. de Gennes, M. Rinaudo, M. Moan, and C. Wolff, J. Polym. Sci., Polym. Lett. Ed. 17, 379 (1979).

${ }^{19}$ K. Kaji, H. Urakawa, T. Kanaya, and R. Kitamaru, Macromolecules 17, 1835 (1984).

${ }^{20}$ M. Nierlich, F. Boué, A. Lapp, and R. Oberthür, Colloid Polym. Sci. 263, 955 (1985).

${ }^{21}$ K. Nishida, K. Kaji, and T. Kanaya, Macromolecules 28, 2472 (1995).

${ }^{22}$ M. Le Bret, J. Chem. Phys. 76, 6243 (1982).

${ }^{23}$ It should be noted that reconsideration is required for the correspondence between $C^{*}$ and $C_{S}^{*}$ when the degree of polymerization $N$ is high. Exactly speaking, $C^{*}$ is defined as the linearly extrapolated crossover point between $q_{\max } \sim C^{1 / 2}$ relation for the typical semidilute regime and $q_{\max }$ $\sim(C / N)^{1 / 3}$ relation for the typical dilute regime. When $N$ is high, the $q_{\max } \sim C^{1 / 2}$ relation, however, begins to fail at some higher concentration than $C^{*}$. This is attributed to the leveling-off effect of the chain extension during dilution. According to the recent experiments (Refs. 3-7), polyion chains do not extend since the increase of the electrostatic persistence length, $b_{e}$ levels off below a certain dilute concentration. The leveling-off effect of the chain extension shifts $C_{S}^{*}$ to a rather higher concentration than $C^{*}$; the relation $q_{\max } \sim C^{1 / 2}$ breaks in its lower concentration. This behavior $\left(C^{*}<C_{S}^{*}\right)$ becomes remarkable especially when the degree of polymerization $N$ exceeds $10^{3}$.

${ }^{24}$ H. Vink, Makromol. Chem. 182, 279 (1982).

${ }^{25}$ H. Hayashi, F. Hamada, S. Suehiro, N. Masaki, T. Ogawa, and H. Miyaji, J. Appl. Crystallogr. 21, 330 (1988).

${ }^{26}$ L. Wang and V. A. Bloomfield, Macromolecules 23, 804 (1990).

${ }^{27}$ The latter transition is more conspicuous than the former one from the viewpoint of phenomenon. The author discussed privately with Joanny and Muthukumar in the opportunity of "Second International Symposium on Polyelectrolytes" at Inuyama Japan in 1998, which phenomenon corresponds to the semidilute/concentrated transition. At that time, we agreed that the former is relevant to the semidilute/concentrated transition and the latter is quite different and new.

${ }^{28}$ F. Oosawa, J. Polym. Sci. 23, 421 (1957).

${ }^{29}$ G. S. Manning, J. Chem. Phys. 51, 924 (1969).

${ }^{30}$ T. Odijk, J. Polym. Sci., Polym. Phys. Ed. 15, 477 (1977).

${ }^{31}$ J. Skolnick and M. Fixman, Macromolecules 10, 944 (1977).

${ }^{32}$ M. Fixman, J. Chem. Phys. 76, 6346 (1982).

${ }^{33}$ R. Koyama, J. Phys. Soc. Jpn. 58, 2062 (1989).

${ }^{34}$ A. L. Kholodenko, Macromol. Theory Simul. 5, 121 (1996).

${ }^{35}$ S. Förster and M. Schmidt, Adv. Polym. Sci. 120, 51 (1995).

${ }^{36} \mathrm{P}$. Egelstaff, An Introduction to the Liquid State (Academic, London and New York, 1967)

${ }^{37}$ E. W. Fischer, Physica A 201, 183 (1993). 